\title{
An investigation into the microflora of heroin
}

\author{
J. McLAUCHLIN, V. MITHANI, F. J. BOLTON, G. L. NICHOLS*, M. A. BELLIS†, Q. SYED†, \\ R. P. M. THOMSON§ and J. R. ASHTON\|
}

PHLS Food Safety Microbiology Laboratory, Central Public Health Laboratory and * Environmental Surveillance Unit, Communicable Disease Surveillance Centre, 61 Colindale Ave, London NW9 5HT, †Birkenhead \& Wallasey Primary Care Trust, St Catherine's Hospital, Birkenhead, Merseyside CH42 OLP, †Communicable Disease Surveillance Centre (North West), Chester CH1 4EF, §South Sefton Primary Care Trust, Burlington House, Crosby Road North, Waterloo, Liverpool L22 OQB and IDepartment of Public Health North West Region, Millennium Park, Birchwood, Warrington WA3 70N

In 2000, an unusual increase of morbidity and mortality among illegal injecting drug users in the UK and Ireland was reported and Clostridium novyi was identified as the likely source of the serious infection, although infections due to $C$. botulinum and Bacillus cereus were also reported. Because heroin was a possibile source of infection, this study investigated the microflora of heroin samples seized in England during 2000 and 2002. Two methods were developed for the examination of the microflora of heroin. The first consisted of suspension of the drug in maximum recovery diluent (MRD) which was inoculated directly into Clostridium Botulinum Isolation Cooked Meat Broth (CBI). The second method rendered the heroin soluble in citric acid, concentrated particulate material (and bacterial cells) by filtration and removed heroin residues by washing with citric acid and phosphate-buffered saline before placing the filter in CBI broth. Duplicate CBI broths from both methods were incubated without heating and after heating at $60^{\circ} \mathrm{C}$ for $30 \mathrm{~min}$. Subcultures were made after incubation for 7 and 14 days on to eight different solid media. The methods were evaluated with heroin samples spiked with either $C$. botulinum or $C$. novyi spore suspensions; recovery of 10 spores in the original sample was demonstrated. Fifty-eight heroin samples were tested by citric acid solubilisation and 34 by the MRD suspension technique. Fifteen different grampositive species of four genera were recognised. No fungi were isolated. Aerobic endospore-forming bacteria (Bacillus spp. and Paenibacillus macerans) were the predominant microflora isolated and at least one species was isolated from each sample. B. cereus was the most common species and was isolated from $95 \%$ of all samples, with $B$. licheniformis isolated from $\mathbf{4 0 \%}$. Between one and five samples yielded cultures of B. coagulans, B. laterosporus, B. pumilus, B. subtilis and P. macerans. Staphylococcus spp. were isolated from $23(40 \%)$ samples; $S$. warneri and $S$. epidermidis were the most common and were cultured from $13(22 \%)$ and $6(10 \%)$ samples respectively. One or two samples yielded cultures of $S$. aureus, $S$. capitis and $S$. haemolyticus. The remainder of the flora detected comprised two samples contaminated with $\boldsymbol{C}$. perfringens and two samples with either $C$. sordellii or $C$. tertium. Multiple bacterial species were isolated from 43 (74\%) samples, a single species from the remaining 15. In 13 samples $B$. cereus alone was isolated, in one $B$. subtilis alone and in one sample $B$. pumilus alone. $C$. botulinum and $C$. novyi were not isolated from any of the heroin samples. Recommendations for the optimal examination of the microflora of heroin are given.

Received 16 Aug. 2002; accepted 26 Aug. 2002. Corresponding author: Dr J. McLauchlin (e-mail: jmclauchlin@phls.org.uk).

\section{Introduction}

Several viral and bacterial infections are associated with illegal injecting drug use [1-3]. Endosporeforming bacteria, including Clostridium botulinum 
[4-6], C. tetani [7] and Bacillus anthracis [8], are increasingly recognised as a group of agents infecting injecting drug users (IDUs). These agents are particularly problematic in this patient group because of their occurrence in the intestinal tract, widespread distribution in the environment, the robust nature of bacterial endosopores and their resistance to the process of drug preparation [9].

In 2000, an unusual increase of morbidity and mortality among IDUs in the UK and Ireland was reported. During the intensive investigation more than 108 cases with at least 43 deaths were recognised between April and Aug. 2000 [10-13]. C. novyi type A was the likely cause of the more serious illnesses [12,14-16], although other endospore-forming bacteria were also associated with some cases including $C$. perfringens [16] and B. cereus [17]. Cases of wound botulism were also reported in IDUs during 2000 and these represent the first recognition of this condition in the UK [18-21].

During the course of the investigations among IDUs during 2000, B. cereus, C. botulinum and C. perfringens were isolated from either heroin or drug injection equipment ([17] PHLS unpublished data). Because of the possibility that heroin was the source of infection, especially with $C$. novyi, investigations were established to develop methods for the analysis of the microbial flora of heroin. There are relatively few data on the microflora of heroin [22-25] and none available for drugs seized in the UK. During drug injection, heroin is solubilised in acidic solutions. Because heroin has been reported to have marked antimicrobial properties [26], methods were devised to culture from both suspensions of the drug as well as solutions made by dissolving the drug in citric acid and attempting to wash away antimicrobial residues by filtration. This report describes the results obtained from the characterisation of the microflora of heroin seized in England during 2000 and 2002.

\section{Materials and methods}

\section{Microbiological media and reagents}

A citric acid (C0706; Sigma-Aldrich) 10\% w/v solution was prepared in distilled water and filter sterilised through a $0.2-\mu \mathrm{m}$ membrane filter into a sterile bottle. This solution was prepared fresh each time of use. Maximum Recovery Diluent (MRD; peptone saline diluent CM733; Oxoid), horse blood 5\% in columbia blood agar base (CBA; Oxoid), Polymyxin Pyruvate Egg Yolk Mannitol Bromothymol Blue Agar (PEMBA, Oxoid), Sabouraud dextrose agar (Sab; CM41; Oxoid), Neomycin Blood Agar (NeoBA; Oxoid) and phosphatebuffered saline (PBS; Dulbecco A phosphate-buffered saline; Oxoid) were prepared according to the manufacturers' instructions.
Clostridium Botulinum Isolation (CBI) Cooked Meat Broth was prepared as described previously [27]. On the day of use, the broths were steamed for $15 \mathrm{~min}$ (with loose lids), cooled to room temperature (with tightened lids) and sterile lysozyme was added to give a final concentration of $10 \mu \mathrm{g} / \mathrm{ml}$.

All agar plates used for anaerobic growth were incubated in either a gas jar with an anaerobic gas generation system (Oxoid) or in an anaerobic cabinet (Don Whitley Scientific) with an atmosphere of $\mathrm{N}_{2}$ $80 \%, \mathrm{H}_{2} 10 \%, \mathrm{CO}_{2} 10 \%$. All agar plates for anaerobic culture were pre-reduced in either an anaerobic jar or an anaerobic cabinet overnight. CBI agar containing egg-yolk emulsion and antibiotic (ACBI) agar containing cycloserine sulphamethoxazole trimethoprim eggyolk emulsion were prepared as described previously [27]. BMRL medium with horse blood (BMBH) was prepared as described elsewhere [28].

\section{Heroin samples}

Samples of heroin seized by the Merseyside Police during 2000 and 2002 were made available for analysis. All samples were received at the Central Public Health Laboratory (CPHL) under police escort and stored securely during the course of this investigation. The samples were taken from items seized as exhibits in criminal prosecutions in the Merseyside area during and after the period of the heroin-related deaths. Heroin analysis was performed for the preservation of evidential integrity. With support from local and regional public health officials and advice from the Crown Prosecution Service, Chief Police Officers for the area agreed to provide samples, and CPHL obtained Home Office authority to be in possession of drugs scheduled as class 1, 2, 3 and 4 under the Misuse of Drugs Act 1971. At the conclusion of this investigation, all material was disposed of under Home Office supervision.

\section{Method 1, suspension in MRD}

A 0.1 -g sample of heroin was suspended in $10 \mathrm{ml}$ of MRD and $0.1 \mathrm{ml}$ was inoculated directly on to two CBA, one PEMBA and one Sab agar plates. Three plates were incubated aerobically at $37^{\circ} \mathrm{C}$ and one of the CBA plates was incubated anaerobically at $30^{\circ} \mathrm{C}$. Two 1-ml samples of the suspensions of heroin in MRD were each filtered through a sterile $0.2-\mu \mathrm{m}$ membrane filter and inoculated into a CBI cooked meat broth. One broth was heated at $60^{\circ} \mathrm{C}$ for $30 \mathrm{~min}$ in a water bath and both were incubated at $30^{\circ} \mathrm{C}$. Heated and unheated broths were subcultured after 7 and 14 days on to pre-reduced ACBI, CBI, NeoBA, BMBH and CBA plates (incubated anaerobically) and CBA incubated aerobically. All plates were incubated at $30^{\circ} \mathrm{C}$, except the NeoBA which was incubated anaerobically at $37^{\circ} \mathrm{C}$. Plates were examined daily for 4 days except for Sab which was examined after 7 days. 


\section{Method 2, citric acid solubilisation}

A 0.1-g sample of heroin was dissolved in citric acid. A minimum of $50 \mathrm{ml}$ was used, although larger volumes were required to dissolve all solid material in some samples. From this solution, $0.1 \mathrm{ml}$ was spread plated on to each of ACBI, CBI, NeoBA, BMBH and CBA plates (which were pre-reduced and incubated anaerobically at $30^{\circ} \mathrm{C}$, except for NeoBA which was incubated at $37^{\circ} \mathrm{C}$ ), and CBA, PEMBA and Sab agar plates which were incubated aerobically at $37^{\circ} \mathrm{C}$. All plates were examined daily for 4 days except Sab agar, which was examined for growth up to 7 days.

Volumes of heroin dissolved in citric acid equivalent to $0.02 \mathrm{~g}$ were filtered through a sterile $0.2-\mu \mathrm{m}$ membrane filter. The filter was rinsed with $20 \mathrm{ml}$ of citric acid, then with $40 \mathrm{ml}$ of PBS. One inoculated filter was placed in each of two pre-reduced CBI cooked meat broths with lysozyme; one of these was heated to $60^{\circ} \mathrm{C}$ for $30 \mathrm{~min}$ in a water bath. Both broths were incubated at $30^{\circ} \mathrm{C}$ and subcultured at 7 and 14 days as described above for the MRD method.

\section{Identification of isolates}

Because of the large numbers of isolates obtained, representatives of each different colonial type from the different treatments were identified.

Bacillus isolates were initially identified on the basis of Gram's and spore stains, colonial morphology and growth on blood agar incubated anaerobically and aerobically. Isolates were identified to species level by methods outlined previously [29-31] in the PHLS Food Safety Microbiology Laboratory.

Lipase and lecithinase reactions of suspected clostridia on CBI plates were recorded. Clostridia were identified as gram-positive (variable) rods, catalase negative, sensitive to metronidazole and growing anaerobically but not aerobically. Isolates suspected to be $C$. perfringens (typical morphology on blood agar and positive Nagler test) were identified in the PHLS Food Safety Microbiology Laboratory. All other anaerobes were identified by the PHLS Anaerobe Reference Unit, Cardiff, as described elsewhere [31].

Isolates of Staphylococcus spp. were identified in the Laboratory of Hospital Infection (CPHL) as described elsewhere [32].

\section{Method validation}

For validation of the methods, spore suspensions of $C$. botulinum and $C$. novyi were prepared. Two $C$. novyi strains were used in these experiments; one was from a case of gas gangrene in 1920 (NCTC 538, National Collection of Type Cultures, CPHL, London) and the second (R14386, Anaerobe Reference Unit, PHLS,
Cardiff) was from the thigh tissue of an IDU case in 2000 [33] which was indistinguishable from other isolates from IDUs during 2000 [34]. A C. botulinum culture from an infected wound of an IDU (R254/02, PHLS FSML, London) isolated in 2002 was also included in this analysis. Spore suspensions were prepared by harvesting the growth from blood agar plates after incubation for 4 weeks under anaerobic conditions. Numbers of viable spores were estimated by colony counts after heat shock at $60^{\circ} \mathrm{C}$ for $30 \mathrm{~min}$ of a decimal dilution series in MRD from which 0.1-ml volumes were spread plated on to pre-reduced blood agar and incubated for 3 days anaerobically at $30^{\circ} \mathrm{C}$.

To ensure the recovery of $C$. botulinum and $C$. novyi from heroin, a heroin sample in MRD or citric acid was 'spiked' with a decimal dilution series of either $C$. botulinum or $C$. novyi spore suspensions. The spiked heroin samples were filtered as described above and inoculated into CBI broths. Spore suspensions were added to give final concentrations of 10-100000 spores in each CBI broth (both heated and unheated) and subcultured on to CBA (incubated aerobically and anaerobically), CBI and ACBI agars. Both C. botulinum and $C$. novyi were recognised by characteristic colonial morphology and positive lipase reaction.

To simulate the possible inhibition of C.botulinum or C.novyi growth in the liquid enrichment broths resulting from co-culture with Bacillus spp., vegetative cells of $B$. licheniformis (growth from two colonies suspended in MRD) and spores of either C. botulinum or $C$. novyi were added to heroin in MRD or citric acid. The original heroin sample was selected to already contain $B$. cereus as part of its microflora. The heroin suspensions or solutions, which had a final C. botulinum or C. novyi concentration of 10-100 spores, were filtered and inoculated into CBI broths, heated or unheated, and then subcultured after 7 and 14 days on to CBA (incubated aerobically and anaerobically) and $\mathrm{CBI}, \mathrm{ACBI}, \mathrm{BMBH}$ and NeoBA agars incubated anaerobically.

\section{Results}

\section{Method validation}

With the first experimental protocol and spiked samples of heroin, viable $C$. botulinum (strain R254/02) and $C$. novyi (R14286) were recovered from enrichment broths with 10-100000 spores (both with and without heating) after either 7 or 14 days. Neither organism was recovered on direct subculture. A similar experiment was performed with C. novyi strain NCTC 538, which was recovered after incubation for 7 days in enrichment broth with both the MRD and citric acid protocols, with and without heating, when 1000 spores were spiked into the original sample.

C. botulinum (strain R254/02) and C. novyi (R14286) 
were detected in the spiking experiment when both $B$. cereus and $B$. licheniformis were present, and were recovered from both heated and unheated broths after 7 and 14 days on all media except the CBA incubated aerobically.

In both experiments, $C$. botulinum and $C$. novyi were not recovered from uninoculated controls.

\section{Microflora of heroin}

A total of 58 heroin samples was analysed. All samples were tested by the citric acid solubilisation technique and 34 by the MRD suspension technique.

On direct inoculation, growth was recorded with only seven samples. Growth was detected only from the MRD suspension; no growth was observed following citric acid solubilisation. Among the seven samples in which bacterial growth was detected after direct inoculation, growth was observed on CBA incubated aerobically only where $1-27$ colonies were recorded; $B$. cereus alone was identified in six samples and $B$. licheniformis alone in one sample.

The list of all bacterial species isolated from the 58 samples by all treatments is shown in Table 1. Fifteen different gram-positive species of four genera were identified. Two isolates (one Bacillus sp. and one Staphylococcus sp.) were non-viable before full identification could be performed and were identified to genus level only. No yeasts or moulds were isolated. Aerobic endospore-forming bacteria (Bacillus spp. and Paenibacillus macerans) were the predominant microflora isolated and at least one of these species was isolated from each sample. B. cereus was the most common bacterium and was isolated from $95 \%$ of samples, followed by B. licheniformis (40\%). The next most common components of the microflora detected were Staphylococcus spp. which were isolated from 23

Table 1. Species of bacteria isolated from 58 samples of heroin

\begin{tabular}{lc}
\hline Species of bacteria & Number of samples $(\%)$ \\
\hline B. cereus & $55(95)$ \\
B. coagulans & $1(2)$ \\
B. licheniformis & $23(40)$ \\
B. laterosporus & $1(2)$ \\
B. pumilus & $5(9)$ \\
B. subtilis & $1(2)$ \\
Bacillus sp. & $1(2)$ \\
C. perfringens & $2(3)$ \\
C. sordellii & $1(2)$ \\
C. tertium & $1(2)$ \\
P. macerans & $1(2)$ \\
S. aureus & $1(2)$ \\
S. capitis & $2(3)$ \\
S. epidermidis & $6(10)$ \\
S. haemolyticus & $1(2)$ \\
S. warneri & $13(22)$ \\
Staphylococcus sp. ${ }^{*}$ & $1(2)$ \\
\hline
\end{tabular}

*Isolates not fully identified.
(40\%) samples. S. warneri and S. epidermidis were the most commonly isolated and were detected in $13(22 \%)$ and $6(10 \%)$ samples, respectively. The remainder of the flora isolated comprised three species of Clostridium (C. perfringens, $C$. sordellii and $C$. tertium) which were isolated from four different samples of heroin. Multiple bacterial species were isolated from 42 (74\%) samples; a single species was isolated from the other 16. B. cereus alone was isolated from 14 of the samples, B. subtilis alone from one and B. pumilus alone from one sample.

The treatment (MRD, citric acid, heated or unheated) and day of isolation for all the bacterial species is shown are Table 2. No growth was detected after either MRD or citric acid treatment in the heated broths from $20-47 \%$ of the samples, but was detected in all except one broth without heating. Seven different bacterial species were isolated after the MRD treatment and 14 after the citric acid treatment. No major differences were detected between the MRD or citric acid treatments for the isolation of Bacillus spp. However, with both the MRD and citrate treatments, B. cereus was more likely to be grown from unheated than heated broths at both 7 and 14 days, and B. licheniformis was more likely to be grown from heated broths at day 7 but not at day 14. C. perfringens and C. sordellii were isolated only from heated broths after both MRD and citric acid treatment. C. tertium was grown from one sample from an unheated broth only after citric acid treatment. Except for two isolates of S. warneri, the remainder of the Staphylococcus isolates were detected only after citric acid treatment in the unheated CBI broths, although $S$. epidermidis and $S$. warneri were isolated from one sample each after heat treatment.

The media with which the different species were isolated are shown in Table 3. B. cereus was isolated on all media, although less often on the anaerobically incubated antibiotic-containing media (i.e., ACBI and NeoBA). The remaining Bacillus spp. and the single $P$. macerans isolate were isolated only from the nonselective media incubated both anaerobically and aerobically, and did not grow on PEMBA, ACBI or NeoBA. The three Clostridium spp. were isolated on all anaerobically incubated media, except $C$. sordellii which was not isolated on CBA or NeoBA, and $C$. tertium which was not isolated on ACBI agar. Staphylococcus spp. were isolated from all media except ACBI and NeoBA.

\section{Discussion}

At the onset of this study, there were limited data on the optimal method for the examination and characterisation of the microflora of heroin. Tuazon and colleagues [22] examined 100 samples of heroin collected in Washington during 1972, Shamsuddin et al. [23] examined 49 samples again from Washington 
Table 2. Treatment and day of isolation of individual bacterial species from 58 samples of heroin

\begin{tabular}{|c|c|c|c|c|c|c|}
\hline \multirow[b]{3}{*}{ Species isolated } & \multicolumn{6}{|c|}{ Number of isolates after } \\
\hline & \multicolumn{3}{|c|}{7 days } & \multicolumn{3}{|c|}{14 days } \\
\hline & Heated & Unheated & Total & Heated & Unheated & Total \\
\hline \multicolumn{7}{|c|}{ MRD suspension method $(\mathrm{n}=34)$} \\
\hline B. cereus & 13 & 30 & 30 & 13 & 31 & 33 \\
\hline B. coagulans & 0 & 0 & 0 & 0 & 0 & 0 \\
\hline B. licheniformis & 4 & 2 & 6 & 5 & 9 & 14 \\
\hline B. laterosporus & 0 & 0 & 0 & 0 & 0 & 0 \\
\hline B. subtilis & 0 & 0 & 0 & 0 & 1 & 1 \\
\hline B. pumilus & 0 & 1 & 1 & 1 & 1 & 2 \\
\hline C. perfringens & 1 & 0 & 1 & 1 & 0 & 1 \\
\hline C. sordellii & 1 & 0 & 1 & 1 & 0 & 1 \\
\hline C. tertium & 0 & 0 & 0 & 0 & 0 & 0 \\
\hline P. macerans & 0 & 0 & 0 & 0 & 0 & 0 \\
\hline S. aureus & 0 & 0 & 0 & 0 & 0 & 0 \\
\hline S. capitis & 0 & 0 & 0 & 0 & 0 & 0 \\
\hline S. epidermidis & 0 & 0 & 0 & 0 & 0 & 0 \\
\hline S. haemolyticus & 0 & 0 & 0 & 0 & 0 & 0 \\
\hline S. warneri & 0 & 2 & 2 & 0 & 2 & 2 \\
\hline No growth & 16 & 0 & 0 & 13 & 1 & 1 \\
\hline \multicolumn{7}{|c|}{ Citric acid solubilisation method $(\mathrm{n}=58)$} \\
\hline B. cereus & 36 & 54 & 54 & 35 & 52 & 55 \\
\hline B. coagulans & 1 & 1 & 1 & 0 & 0 & 0 \\
\hline B. licheniformis & 10 & 5 & 15 & 13 & 12 & 25 \\
\hline B. laterosporus & 0 & 0 & 0 & 1 & 1 & 1 \\
\hline B. subtilis & 0 & 0 & 0 & 0 & 0 & 0 \\
\hline B. pumilus & 1 & 1 & 2 & 2 & 5 & 5 \\
\hline C. perfringens & 1 & 0 & 1 & 1 & 0 & 1 \\
\hline C. sordellii & 1 & 0 & 1 & 1 & 0 & 1 \\
\hline C. tertium & 0 & 1 & 1 & 0 & 1 & 1 \\
\hline P. macerans & 1 & 0 & 1 & 0 & 0 & 0 \\
\hline S. aureus & 0 & 1 & 1 & 0 & 1 & 1 \\
\hline S. capitis & 0 & 1 & 1 & 0 & 2 & 2 \\
\hline S. epidermidis & 1 & 2 & 3 & 1 & 6 & 6 \\
\hline S. haemolyticus & 0 & 1 & 1 & 0 & 1 & 1 \\
\hline S. warneri & 2 & 8 & 10 & 4 & 9 & 13 \\
\hline No growth & 12 & 0 & 0 & 13 & 0 & 0 \\
\hline
\end{tabular}

Table 3. Media used to recover different species of bacteria from 58 samples of heroin

\begin{tabular}{|c|c|c|c|c|c|c|c|}
\hline \multirow[b]{2}{*}{ Species } & \multicolumn{7}{|c|}{ Number of samples with isolation on } \\
\hline & Total & $\mathrm{CBA}$ & PEMBA & CBI & $\mathrm{ACBI}$ & $\mathrm{BMBH}$ & NeoBA \\
\hline B. cereus & 55 & 55 & 33 & 55 & 28 & 55 & 10 \\
\hline B. coagulans & 1 & 1 & 0 & 1 & 0 & 1 & 0 \\
\hline B. licheniformis & 23 & 23 & 0 & 23 & 0 & 23 & 0 \\
\hline B. laterosporus & 1 & 1 & 0 & 1 & 0 & 1 & 0 \\
\hline B. pumilus & 5 & 5 & 0 & 4 & 0 & 0 & 0 \\
\hline B. subtilis & $1^{*}$ & 0 & 0 & 0 & 0 & 0 & 0 \\
\hline C. perfringens & 2 & 2 & 0 & 2 & 2 & 2 & 2 \\
\hline C. sordellii & 1 & 0 & 0 & 1 & 1 & 1 & 0 \\
\hline C. tertium & 1 & 1 & 0 & 1 & 0 & 1 & 1 \\
\hline P. macerans & 1 & 1 & 0 & 1 & 0 & 1 & 0 \\
\hline S. aureus & 1 & 1 & 0 & 0 & 0 & 1 & 0 \\
\hline S. capitis & 2 & 2 & 1 & 2 & 0 & 0 & 0 \\
\hline S. epidermidis & 6 & 6 & 0 & 5 & 0 & 4 & 0 \\
\hline S. haemolyticus & 1 & 1 & 0 & 0 & 0 & 0 & 0 \\
\hline S. warneri & 13 & 13 & 4 & 9 & 0 & 13 & 0 \\
\hline
\end{tabular}

PEMBA, polymyxin pyruvate egg-yolk mannitol bromothymol blue agar (aerobic); CBI, Clostridium Botulinum Isolation Agar; ACBI, Antibiotic Clostridium Botulinum Isolation Agar; $\mathrm{BMBH}, \mathrm{BMRL}$ agar with horse blood; NeoBA, neomycin blood agar (all anaerobic).

* Isolated from aerobically incubated CBA only.

in 1981 and Moustoukas et al. [25] examined 31 samples obtained in New Orleans during 1979-1980. The methods used were direct plating together with adding heroin directly to thioglycolate [22], trypticase soy [23] or chopped meat glucose [25] broths and subculture on to solid selective media after incubation for 4-14 days. Antimicrobial activity of heroin was noted, especially against staphylococci $[25,26]$. Two 
methods were developed in the present study for analysis of the microflora of heroin. One consisted of suspending heroin in MRD broth, which was inoculated directly into a CBI broth. The second method consisted of solubilising the drug in citric acid and recovering insoluble residues (including bacterial cells) free of heroin by filtration and washing with further volumes of citric acid and then PBS. The results presented here are consistent with antibacterial activities of heroin in that more bacterial species (especially the staphylococci) were isolated by the citric acid solubilisation technique than by suspension of the drug in MRD. This is despite the fact that the MRD procedure described here used five times more heroin from each sample than the citric acid method. None of the previous studies $[22,23,25]$ used heating as a selective procedure, which also has the effect of heat-shocking (activating) bacterial endospores. The present study demonstrated the usefulness of this technique by the isolation of the small numbers of clostridia, although treatment to higher temperatures $\left(70-80^{\circ} \mathrm{C}\right)$ has been advocated by others for the isolation of this group of organisms [35].

Among the 100 heroin samples examined in the USA in 1972 [22], at least one micro-organism was isolated from $89 \%$ of samples, $44 \%$ as a single organism only. The four most common components of the microflora of the heroin examined in the USA were Bacillus spp. from $29 \%$ of the samples, Aspergillus spp. from $24 \%$, coagulase-negative staphylococci from $17 \%$ and $C$. perfringens from $10 \%$ [22]. Similar results were obtained in the two other studies in the USA $[23,25]$, in which no organisms were recovered from $39 \%$ and $69 \%$ of samples and bacteria (Bacillus spp. plus a small number of gram-positive cocci) and fungi (Aspergillus and Penicillium spp.) were the only organisms isolated. This is similar to the data presented here for the range of organisms isolated, with the exception of fungi, which were not isolated from any samples examined here. However, in this study, all samples were shown to be contaminated with at least one species of Bacillus ( $B$. cereus followed by $B$. licheniformis were the most common), $40 \%$ of samples contained a Staphylococcus sp. (almost all of which were coagulase-negative) and a small number of other species (P. macerans, C. perfringens, C. sordellii and C. tertium) were also isolated. More than one bacterial species was isolated from $74 \%$ of the samples examined here. Although information is not available to categorise the type of heroin previously examined, a similar but more extensive microflora was detected here than described previously [22], suggesting advantages of the methods described here.

Based on the data presented here, subculturing at 7 and 14 days is necessary because some organisms were isolated only on one of these occasions, and no additional advantages were found from cultivation on $\mathrm{BMBH}$ or NeoBA agars. No additional advantage was observed over the incubation temperature of $37^{\circ} \mathrm{C}$ used for the NeoBA.

A limited range of both genera and species was isolated from the heroin samples described here. Although this may reflect a limited microflora that survives in heroin, it may also reflect interactions between organisms during in-vitro cultivation which prevent recovery. The ubiquitous presence of Bacillus spp. in all samples makes recovery of anaerobic organisms more difficult, even on solid media with antibiotics. Because, with the exception of the effect of heating broths, there are no selective media for isolation of C. botulinum or C. novyi, growth of the heroin microflora in the non-selective broths may inhibit the growth of these organisms, although this was not demonstrated in 'spiking' experiments. The presence of a single Bacillus sp. in $26 \%$ of the samples is probably indicative of interactions within the nonselective broths. The possible inhibitory effects of the heroin are discussed above.

Although no direct quantitative data were obtained, the results from direct examination of the heroin described here suggest that small numbers of bacteria are present in the heroin. Heroin is prepared from opium, which is a dried latex obtained from the opium poppy. Morphine base is extracted from opium and, as this is insoluble it cannot be injected and is converted to heroin by a chemical process involving heat and treatment with acetic anhydride. Adulterants are added to heroin during processing and cutting. A limited range of microflora was detected both here and previously [22], comprising both aerobic and anaerobic endosporeforming bacteria (i.e., those belonging to the genera Bacillus, Clostridium and Paenibacillus) and staphylococci. Endospore-forming bacteria are all present in soil in the environment; some of them also occur in the intestinal tracts of man and animals. Hence, these are likely to be present in the original plant material, as well as in the heroin manufacturing environment and in any adulterants. It is not known if the endosporeforming bacteria survive the heroin manufacturing process, but as manufacture is performed under nonsterile conditions, post process contact with the environment is just as likely a source of contamination as the original starting material. Although robust, staphylococci do not produce endospores and are generally not as environmentally resistant as members of the genera Bacillus and Clostridium. All five species of Staphylococcus detected (i.e., S. aureus, S. capitis, $S$. epidermidis, $S$. haemolyticus and $S$. warneri) are primarily resident on human skin (and sometimes that of other primates), with more temporary residence on domestic artiodactyls (pigs and horses) reported for $S$. haemolyticus and S. warneri [32]. Therefore, one likely source of contamination of this heroin is direct contact with the skin flora of the producers or processors.

During the course of the investigation of illness among 
IDUs in 2000, 16 samples of heroin were collected in England and Scotland (two of which were not identified as part of the outbreak) and examined by various techniques $[16,17,34,35]$. One of the samples was obtained from an IDU and indistinguishable isolates of $B$. cereus were obtained from both the aspirate from an injection site wound and from a heroin sample used by the patient $[17,34]$. A summary of the results from these 16 samples is given in Table 4 . Examination of syringe residues during 2000 also yielded cultures of $C$. perfringens $[16,34]$ and $C$. botulinum types A and B [35]. Results from that investigation (Table 4), together with the 58 samples described in this study did not identify any samples of heroin contaminated with either $C$. novyi or $C$. botulinum despite the fact that spiking experiments demonstrated that the procedures described here were capable of recovering these bacteria. Because only very low levels of all bacteria were detected, the possibility that $C$. novyi and $C$. botulinum were both present but were below the detection limit of the method used here cannot be excluded. However, these studies did identify contamination of heroin by $B$. cereus, $C$. perfringens and $S$. aureus, all of which were isolated from clinical samples collected from infected IDUs during the 2000 outbreak $[16,17]$. B. cereus endocarditis and ophthalmitis from contaminated heroin or injection paraphernalia have been reported elsewhere [23,36]. Staphylococcal endocarditis is a well recognised complication of injecting drug use [37]. The sources of $C$. perfringens and $S$. aureus for infection in IDUs are probably more likely to be the patients' own gut and skin flora. However, one case of $B$. cereus infection, described above, was associated with a contaminated sample of heroin [17]. Other sources of C. novyi or C. botulinum, such as injection paraphernalia or the acidulates used during drug injection, were not investigated. Lemon juice (also used as an acidulant in heroin abuse) has been implicated as a source of Candida albicans in outbreaks of systemic candidosis among IDUs [38, 39].

Table 4. Results on the examination of 18 samples of heroin collected during 2000 and examined outside this study

\begin{tabular}{lc}
\hline Species of bacteria & Number of samples \\
\hline B. cereus $^{*}$ & 4 \\
B. firmus & 1 \\
B. licheniformis & 7 \\
Bacillus spp. & ${ }^{\dagger}$ \\
C. bifermentans & 2 \\
C. perfringens & 1 \\
C. saccharolyticum & 2 \\
C. sporogenes & 1 \\
Staphylococcus sp. ${ }^{\dagger}$ & 1 \\
\hline
\end{tabular}

Sixteen of the samples were collected in England and Scotland during the course of the investigation of illness among IDUs during $2000[16,34,35]$, two samples (plus wrapping for one sample) were unrelated to this outbreak.

${ }^{*}$ One B. cereus isolate directly linked to infection in a heroin user [17].

${ }^{\dagger}$ Isolates not fully identified.
In summary, an optimised and evaluated protocol for the examination of the microflora of heroin is described. The method involves the use of citric acid solubilisation followed by filtration, washing with citric acid and PBS then inoculation of the filters into CBI broths with and without heat treatment. Broths should be subcultured after 7 and 14 days on to CBA (incubated aerobically and anaerobically), PEMBA (incubated aerobically), $\mathrm{CBI}$ and ACBI (incubated anaerobically). All media should be incubated at $30^{\circ} \mathrm{C}$.

We acknowledge the co-operation of Merseyside Police Drugs Support Team for release and transport of heroin samples which made this study possible, especially to B. Jones and P. Johnson. We also acknowledge the technical assistance of Mr J. Shah, Dr M. Brett and Mr D. Conway (PHLS Central Public Health Laboratory) and Dr C. Beynon (John Moores University, Liverpool). Identification of anaerobic bacteria was performed by Dr J. Brazier (PHLS Anaerobe Reference Unit, Cardiff) and identification of staphylococci by $\mathrm{Dr} \mathrm{T}$. Pitt (PHLS Laboratory of Hospital Infection, Central Public Health Laboratory). We also acknowledge helpful discussion with Dr M. White (Forensic Science Service, London).

\section{References}

1. Horsburgh CR, Anderson JR, Boyko EJ. Increased incidence of infections in intravenous drug users. Infect Control Hosp Epidemiol 1989; 10: 211-215.

2. Brettle RP. Infection and injection drug use. J Infect 1992; 25: 121-131.

3. Peat M, Budd J, Burns SM, Robertson R. Audit of blood borne virus infections in injecting drug users in general practice. Commun Dis Public Health 2000; 3: 244-246.

4. Martin C, Schaller MD, Lepori M, Liaudet L. Cranial nerve palsies and decending paralysis in a drug abuser resulting from wound botulism. Intensive Care Med 1999; 25: 765-769.

5. Werner SB, Passaro D, McGee J, Schechter R, Vugia DJ. Wound botulism in California, 1951-1998: recent epidemic in heroin injectors. Clin Infect Dis 2000; 31: 1018-1024.

6. Jensenius M, Lovstad RZ, Dhaenens G, Rorvik LM. A heroin user with a wobbly head. Lancet 2000; 356: 1160.

7. Talan DA, Moran GJ. Tetanus among injecting drug users: California 1997. Ann Emerg Med 1998; 32: 385-386.

8. Ringertz SH, Hoiby EA, Jensenius $\mathrm{M}$ et al. Injectional anthrax in a heroin skin- popper. Lancet 2000; 354: 1574-1575.

9. Bloomfield SF, Arthur M. Mechanisms of inactivation and resistance of spores to chemical biocides. Soc Appl Bacteriol Symp Suppl 1994; 23: 91S-104S.

10. Christie B. Gangrene bug "killed 35 heroin users". BMJ 2000; 320: 1690.

11. Anon. Serious unexplained illness among drug injectors. Commum Dis Rep CDR Wkly 2000; 24: 195.

12. Anon. Clostridium novyi is likely cause of 'serious unexplained illness' as cases continue to be reported. Commum Dis Rep CDR Wkly 2000; 24: 213, 216.

13. Bellis MA, Beynon C, Millar T et al. Unexplained illness and deaths among injecting drug users in England: a case control study using Regional Drug Misuse Databases. J Epidemiol Community Health 2001; 55: 843-844.

14. Anon. Update: Clostridium novyi and unexplained illness among injecting drug users: Scotland, Ireland and England April-June 2000. MMWR Morb Mortal Wkly Rep 2000; 49: $543-544$.

15. Ryan JM, Paul J, Curtis S, Patel NK. Clostridium novyi infection: a fatal association with injecting drug users. Emerg Med J 2001; 18: 138-139.

16. McGuigan CC, Penrice GM, Black M et al. Outbreak of infection with Clostridium novyi and other sporulating bacteria in Scottish injecting drug users. J Med Microbiol 2002; 51: 971-977.

17. Dancer SJ, McNair D, Finn P, Kolstot AB. Bacillus cereus cellulitis from contaminated heroin. J Med Microbiol 2002; 51: $278-281$.

18. Athwal BS, Gale AN, Brett MM, Youl BD. Wound botulism in UK. Lancet 2000; 356: 2001-2002. 
19. Khan M, Chay S, Bonner S, Brett M. Wound botulism in injecting drug users: second case in England. Eurosurveillance Weekly 2000; 22 June.

20. Hood J, Horne G, Sweeney G, Baird K. Third case of wound botulism in an injecting drug user. SCIEH Weekly Report 2000; 34: 200.

21. Mulleague L, Bonner SM, Samuel A et al. Wound botulism in drug addicts in the United Kingdom. Anaesthesia 2001; 56: $120-123$.

22. Tuazon CU, Hill R, Sheagren JN. Microbiological study of street heroin and injection paraphernalia. J Infect Dis 1974; 129: $327-329$

23. Shamsuddin D, Tuazon CU, Levy C, Curtin J. Bacillus cereus panophthalmitis: source of organism. Rev Infect Dis 1982; 4 97-103.

24. Alvarez M, Barturen B, Regulez P, Ponton J, Ezquerra JL, Cisterna R. Microbiological study of 30 samples of heroin. Enferm Infecc Microbiol Clin 1990; 8: 231-233.

25. Moustoukas NM, Nichols RL, Smith JW, Garey RE, Egan RR. Contaminated street heroin. Relationship to clinical infections. Arch Surg 1983; 118: 746-749.

26. Tuazon CU, Miller H, Shamsuddin D. Antimicrobial activity of street heroin. J Infect Dis 1980; 142: 944.

27. Mills DC, Midura TF, Aron SS. Improved selective medium for the isolation of lipase positive Clostridium botulinum from feces of human infants. J Clin Microbiol 1985; 21: 947-950.

28. Barr GD, Hudson MJ, Priddle JD, Jewell DP. Colonic bacterial proteases to $\operatorname{IgA} 1$ and $\operatorname{sgA}$ in patients with ulcerative colitis. Gut 1987; 28: 186-189.

29. Parry JM, Turnbull PCB, Gibson JR. A colour atlas of Bacillus species. London, Wolfe Medical Publications. 1983.

30. Gordon RE, Haynes WC, Pang CHN. The genus Bacillus. Agricultural Research Station Agriculture Handbook 427
Washington, DC, US Department of Agriculture. 1973.

31. Barrow GI, Feltham RKA. Cowan and Steel's manual for the identification of medical bacteria, 3rd edn. Cambridge, Cambridge University Press. 1993.

32. Kloos W. Taxonomy and systematics of Staphylococci indigenous to humans. In: Crossley KB, Archer GL (eds) The staphylococci in human diasease. New York, Churchill Livingstone. 1997: 113-137.

33. McGuigan C, Roworth M. Clostridium novyi type A infection: a sporadic fatal case. Scand J Infect Dis 2002; 34: 209-211.

34. McLauchlin J, Salmon J, Ahmed S et al. Amplified fragment length polymorphism (AFLP) analysis of Clostridium novyi, $C$. perfringens and Bacillus cereus isolated from injecting drug users during 2000. J Med Microbiol 2002; 51: 990-1000.

35. Brazier JS, Duerden BI, Hall $\mathrm{V}$ et al. Isolation and identification of Clostridium spp. from infections associated with the injection of drugs: experiences of a microbiological investigation team. J Med Microbiol 2002; 51: 985-989.

36. Tuazon CU, Murray HW, Levy C, Solny MN, Curtin JA, Sheagren JN. Serious infections from Bacillus spp. JAMA 1979; 241: 1137-1140.

37. Ing MB, Baddour LM, Bayer AS. Bacteremia and infective endocarditis: pathogenesis, diagnosis and complications. In: Crossley KB, Archer GL (eds) The staphylococci in human disease. New York, Churchill Livingstone. 1997: 331-354.

38. Newton John HF, Wise K, Looke DFM. Role of lemon in disseminated candidiasis of heroin abusers. Med J Aust 1984; 140: $780-781$.

39. Podzamczer D, Ventin M, Sauca G, Fernandez-Viladrich P, Martin R, Gudiol F. Contaminated lemons as possible source of infection in heroin abusers with disseminated candidiasis. Eur J Clin Microbiol 1986; 5: 477. 